\title{
Imaging spectrometry for Earth observations
}

Imaging spectrometry is defined as the simultaneous acquisition of images in a large number of contiguous spectral bands. Today, several airborne imaging spectrometers are operational, and in the beginning of the next decade, the High Resolution Imaging Spectrometer will provide data from orbit. This is a facility instrument that is slated for flight on the second series of the National Aeronautics and Space Administration's Earth Observing System platforms. The instrument is designed to acquire 24-kilometer wide, 30-meter pixel images in 192 spectral bands simultaneously in the 0.4-2.45micrometer wavelength region. By the use of pointing mirrors, it will be able to sample any place on Earth, except the poles, every 2 days. It will operate at the intermediate scale between the human and the global and, therefore, will link studies of the Earth's surface processes to the global monitoring that is carried out by lower resolution instruments. So far, over 50 science data products from images of this instrument have been identified in the fields of atmospheric gases, clouds, snow and ice, water, vegetation, and rocks and soils. The key attribute of imaging spectrometry that makes it possible to derive quantitative information from the data is the large number of contiguous spectral bands. Therefore, spectrum matching techniques can be applied. Such techniques are not possible by using today's multispectral scanner data.

\section{Introduction}

Imaging spectrometry for Earth observations has been developed
over the last decade in order to provide more definitive and quanti-
tative information from remote sensing about the surface of the
Earth. The space-based multispectral scanners, USA's Landsat and
France's SPOT, showed many of the advantages of the perspective
from space. The short-wavelength infrared bands of the Landsat
Thematic Mapper (TM) have yielded information about the Earth's
surface, but in many instances, the information is ambiguous as a
result of the small number of spectral bands available. Because the
emphasis now is being placed on global measurements in an effort to
understand global change, more sophisticated sensors are necessary
in order to provide the quantitative information that is important to
the development of predictive models. Imaging spectrometry has the potential to acquire all the information that is available in the radiance reflected from the Earth's surface.

Imaging spectrometry is defined as the simultaneous acquisition of image data in hundreds of contiguous spectral bands, which makes it possible to produce laboratory-like reflectance spectra for each pixel in the image, as shown in figure 1 (Goetz and others, 1985). The High Resolution Imaging Spectrometer. slated for flight on the second of the National Aeronautics and Space Administration's (NASA) Earth Observing System's (EOS-A) series of platforms, will have 192 spectral bands covering the wavelength region of $0.4-2.45$ $\mu \mathrm{m}$.

\section{Background}

Landsat 1, launched in 1972, provided the first multispectral images that could be calibrated radiometrically and that were provided in digital form. The spectral bands were designed primarily for use in agricultural studies. However, the geological community rapidly took advantage of the synoptic view. as well as the spectral coverage that extended beyond the visible part of the spectrum. The need to understand the spectral reflectance characteristics of natural surfaces hastened the development of portable spectrometers that would aid in interpreting Landsat Multispectral Scanner (MSS) images and would provide a fundamental data set for developing the requirements of more sophisticated spectral imaging systems. One of the early instruments was the Portable Field Reflectance Spectrometer (PFRS) (Goetz and others, 1975). Its spectra and laboratories' spectra were reproduced in a series of papers during the 1970s that were summarized by Hunt (1980), and these papers made it clear that direct mineral identification would be possible if imaging was possible in narrow, contiguous spectral bands. Field spectrometry and multiband airborne imaging produced the rational for the seventh band $(2.08-2.36 \mu \mathrm{m})$ on the TM (Abrams and others, 1977).

Although imaging spectrometry was not feasible at that time. the next step beyond field spectrometry was airborne and spaceborne profiling in many spectral bands simultaneously. Two instruments were developed during that period. In 1976 work was begun on the Shuttle Multispectral Infrared Radiometer (SMIRR), a 10-band spinning filter wheel radiometer that flew with the second flight of the space shuttle in 1981. Data from this instrument provided the first direct identification of limestone and the clay kaolinite from orbit (Goetz and others, 1982).

In the late $1970 \mathrm{~s}$, an airborne 500-band profiler was developed by Geophysical Environmental Research in Millbrook, New York, USA, and was used to produce mineralogical maps, as well as to identify trees that were stressed by excess copper in the soil. This profiler led to the discovery of the "blue shift" in the red edge of the chlorophyll absorption feature at $0.68 \mu \mathrm{m}$ (Collins and others, 1983).

In 1981 an imaging spectrometer program was begun at NASA's Jet Propulsion Laboratory in Pasadena, California, USA. This program had as its goal two different airborne imaging spectrometer systems, a shuttle experiment and finally a free-flying version known as the High Resolution Imaging Spectrometer (HIRIS). The first airborne system was the Airborne Imaging Spectrometer (AIS) (Vane and Goetz, 1988), which was, in fact, a detector test 


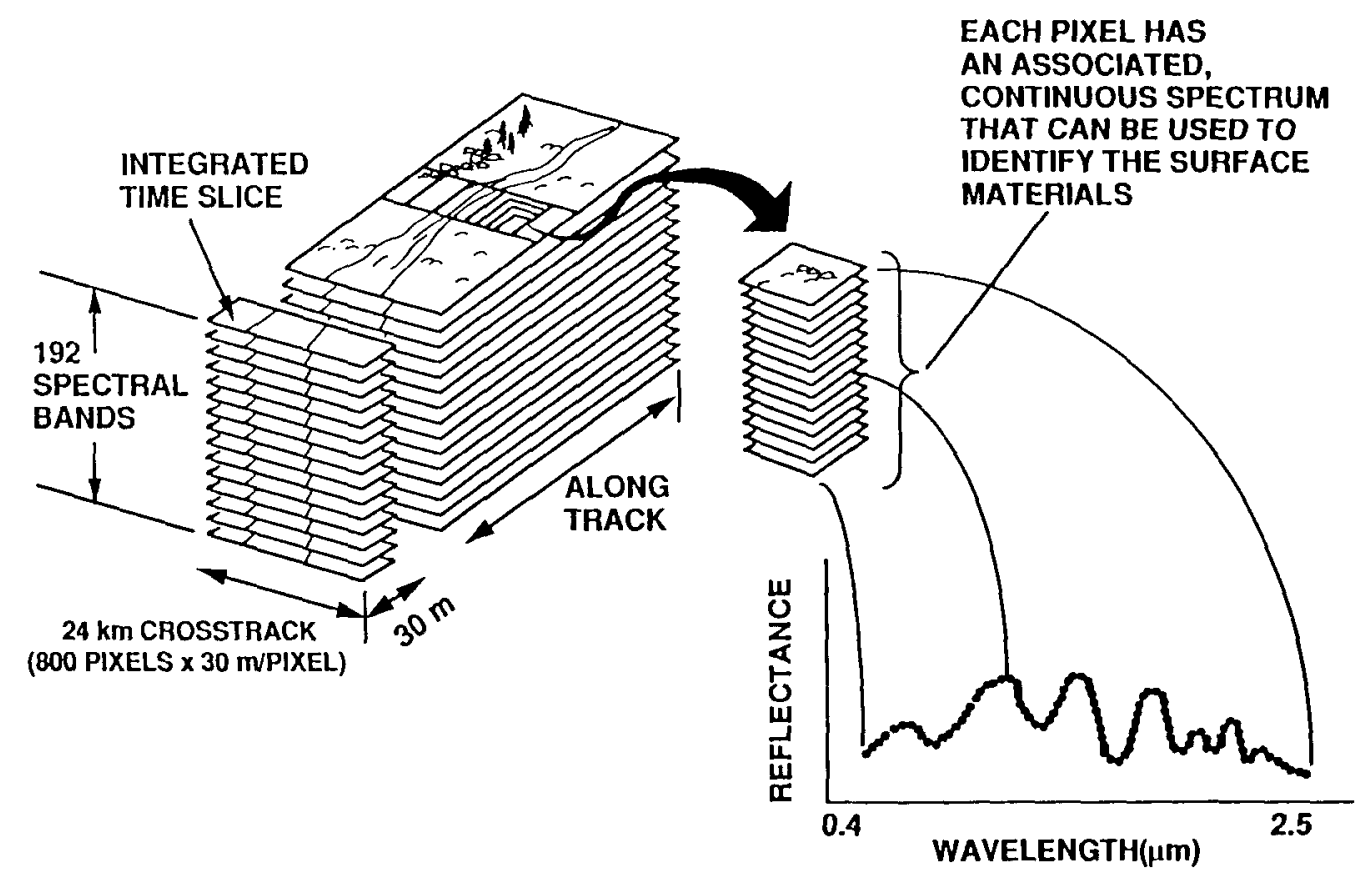

Figure 1.-Concept of imaging spectrometry.

bed. It covered $128,9.3-\mathrm{nm}$ bands in the $1.2-2.4 \mu \mathrm{m}$ region. The spatial swath coverage was only 32 and later 64 picture elements. A coincident development was a new portable spectrometer known as the Portable Instant Display and Analysis Spectrometer (PIDAS) (Goetz, 1987) that recorded a complete spectrum from 0.4 to $2.5 \mu \mathrm{m}$ in 2 seconds. The second airborne system was the 224-band Airborne Visible and Infrared Imaging Spectrometer (AVIRIS) that flies at 20-km altitude on the NASA ER-2 aircraft (Porter and Enmark, 1988). AVIRIS is an optomechanical whiskbroom scanner that has a 1 -milliradian (mrad) instantaneous field of vision (20-m pixels). AVIRIS is operational now and provides unique imaging spectrometry data for atmosphere, land, and water studies.

In 1988 NASA announced that HIRIS would be a facility instrument as part of the EOS package aboard the Polar-Orbiting Platform. The 14-member HIRIS team (table 1) was chosen in 1989. In early 1991, NASA confirmed that HIRIS would be part of the second EOS-A platform scheduled to be flown in 2003 .

\section{Rationale for HIRIS}

HIRIS will embody both high spatial resolution (30-m pixels) and contiguous spectral coverage in the region of $0.4-2.45 \mu \mathrm{m}$ in 192 bands. In addition, HIRIS is designed to point $\pm 45^{\circ}$ crosstrack and +56 to $-30^{\circ}$ downtrack in order to provide coverage of every place on the globe, except the poles, within 2 days and to create bidirectional reflectance distribution functions. HIRIS is a sampling instrument and is not intended to provide global coverage.

HIRIS will operate at the intermediate scale between the human and the global, and thus it is essential for linking the studies of processes at the surface of the Earth to the global monitoring program that is a primary function of EOS. Some of the fundamental questions in Earth system science revolve around the scale dependence of some processes and the scale invariance of others, as well as the interactions of processes that occur at fundamentally different scales. Experimental measurements can investigate processes on the detailed
Table 1.-HIRIS science team members and their affiliations

\begin{tabular}{ll}
\hline John Aber & Hugh Kieffer \\
University of New Hampshire & U.S. Geologial Survey \\
Kendall L. Carder & Flagstaff, Arizona \\
University of South Florida & David A. Landgrebe \\
Roger N. Clark & Purdue University \\
U.S. Geological Survey & John M. Melack \\
Denver, Colorado & University of California, \\
Curtiss O. Davis & Santa Barbara \\
Jet Propulsion Laboratory, & Lawrence C. Rowan \\
California Institute of & U.S. Geological Survey \\
Technology & Reston, Virginia \\
Jeffrey Dozier & Susan L. Ustin \\
University of California, & University of California, \\
Santa Barbara, and Goddard & Davis \\
Space Flight Center & Ronald M. Welch \\
Siegfried A.W. Gerstl & South Dakota School of \\
Los Alamos National & Mining and Technology \\
Laboratory & Carol A. Wessman \\
Alexander F.H. Goetz, & University of Colorado \\
team leader & \\
University of Colorado & \\
\hline
\end{tabular}

level at which the transport of nutrients, sediments, gases, and solutes occurs, such as the uptake of carbon dioxide and nitrogen by plants, the release of water vapor in organic compounds by plants, the movement of sediment in rivers. and the elution of chemicals from the seasonal snowpack. At a scale of hundreds of meters to kilometers, we can examine the large-scale effect of some of these processes, but we cannot investigate scale dependency or address how results from field investigations can be extrapolated to the global scale. Experience has shown that it is logistically infeasible to mount 
an aircraft program for acquiring large amounts of intermediate-scale data. Therefore. HIRIS is essential in order to understand the processes that are taking place within the coarser resolution elements of the Moderate Resolution Imaging Spectrometer (MODIS), another EOS-A instrument, and that are necessitated by the global monitoring function.

HIRIS will permit the detailed examination and monitoring of sensitive ecosystem interfaces where the rates of change are highest and. therefore, most easily detected. Changes are detected best at interfaces, such as the montane snowline, the savanna-woodland border, the wetland-upland edge, and the estuarine-oceanic zone. Many of the important fluxes occur at spatial scales that are far too small to be seen by the coarser resolution mapping instruments or that require high spectral resolution for their detection. Thus a global sampling program emploving HIRIS will be an essential component for a global mapping and modeling program that has instruments designed for large-scale monitoring.

\section{HIRIS science requirements}

The science requirements for HIRIS have been developed by the HIRIS science team since 1989 (Goetz and others, 1991). The science requirements have driven the instrument design, and the resulting major HIRIS parameters are given in table 2. Following is the underlying rationale for some of the major HIRIS functional instrument parameters.

As discussed above. HIRIS acts as an intermediate-level data collection system in a multistage sampling program. Keeping this mission in mind and basing our decisions on the experience of the Landsat TM, we chose a spatial resolution equivalent to that of TM, a ground instantaneous field of view of $30 \mathrm{~m}$. The requirement for a $30-\mathrm{m}$ or smaller pixel can be quantified in vegetation research. In forest ecosystems, successional changes in vegetation structure and function are linked to the size of the gaps that are created by trec death. windfall. and other forms of disturbance. Similarly, arguments can be made for the requirement for high spatial resolution in geological mapping because. in many regions, rock outcrops may have only a few square meters of surface exposure. Alternatively, studies of macrophytes in inland waters, as well as in coastal areas, may require higher spatial resolution than is provided by the $30-\mathrm{m}$ pixel. However, the penalty paid for higher spatial resolution in the form of increased data rate and reduced number of spectral bands was too high. and the $30-\mathrm{m}$ ground instantaneous field of view was established as a requirement.

Table 2. - HIRIS functional instrument parameters

\begin{tabular}{ll}
\hline Item & Performance \\
\hline Design altitude & $705 \mathrm{~km}$ \\
Ground instantaneous field of view & $30 \mathrm{~m}$ \\
Swath width & $24 \mathrm{~km}$ \\
Spectral coverage & $0.4-2.45 \mu \mathrm{m}$ \\
Average spectral sample interval & \\
$\quad 0.4-1 \mu \mathrm{m}$ & $9.4 \mathrm{~nm}$ \\
$\quad 1-2.45 \mu \mathrm{m}$ & $11.7 \mathrm{~nm}$ \\
Pointing & $+56^{\circ}$ to $-30^{\circ}$ \\
$\quad$ Along-track & $+45^{\circ}$ to $-45^{\circ}$ \\
$\quad$ Crosstrack & $12 \mathrm{bits} / \mathrm{pixel}$ \\
Encoding & $405 \mathrm{Mbps}$ \\
Maximum internal data rate & $100 \mathrm{Mbps}$ \\
Maximum output data rate & 1 (off), 2, 3, 4, and 8 \\
Image motion compensation &
\end{tabular}

The choice of spectral coverage was dictated both by scientific requirements and technological capabilities. The $0.4-2.45 \mu \mathrm{m}$ region contains all the information that can be derived by passive sensors from reflected solar energy. In the visible and near-infrated spectral region, diagnostic information is attainable for water. snow, and vegetation and for some minerals and soils. In the region beyond 1 $\mu \mathrm{m}$, diagnostic spectral features are found for snow. clouds, vegetation, and minerals in soils.

The requirements for spectral resolution are driven by the water and vegetation studies in the visible and near-infrared region and by the needs for vegetation biochemistry and mineralogical mapping in the region beyond $1 \mu \mathrm{m}$. Deriving the biochemical constituents from vegetation also requires data over the full spectral range. One of the advances in our understanding biological activity in surface waters by the use of imaging spectrometry will be our ability to identify directly the constituents in their respective concentrations. Absorption spectra of algal pigments, such as phycoerthyrin and peridinin, exhibit features that have [ull-width, one-half-maximum values of $20 \mathrm{~nm}$. Therefore, a $20-\mathrm{nm}$ resolution and $10-\mathrm{nm}$ sampling will be required in order to discern these features in the $0.4-0.7 \mu \mathrm{m}$ region.

Requirements of the spectral resolution for vegetation research are being developed. The resolution offered by TM clearly has not proven sufficient for species identification or for measuring the biochemical state of terrestrial ecosystems beyond the general measurements of stress. One goal of imaging spectrometer research is to develop the capability to detect subtle changes in ecosystem biogeochemistry as precursor indicators of future changes in structure and function. This problem is being studied by the remote sensing of changes in the total canopy content of important constituents such as nitrogen and lignin (Wessman and others, 1988). Advances have been made in extracting spectral features that are associated with dry leaf matter from the spectra of healthy. turgid vegetation (Goetz and others. 1990). In both cases, an approximate 10-nm spectral sampling was adequate for resolving the relevant spectral features.

Spectral features for minerals are associated both with an electronic transition in transition clements, mainly iron, in the visible and near-infrared region and with vibrational modes in the region beyond $1.5 \mu \mathrm{m}$. Vibrational features, generally overtone bending-stretching vibrations, are exhibited by minerals bearing $\mathrm{Al}-\mathrm{OH}, \mathrm{Mg}-\mathrm{OH} \cdot \mathrm{CO}_{3}$. and $\mathrm{H}_{2} \mathrm{O}$ constituents (fig. 2). These minerals have features in some cases as small as 10 -nm full-width, one-half-maximum values that require 5-nm sampling for proper description. However, the majority of mineral features can be described completely by the use of 10 -nm sampling. A significant degradation in identification, particularly in magnesium-bearing minerals, as well as in limestone and dolomite. is seen when the sampling interval is increased to $20 \mathrm{~nm}$. In general. $10 \mathrm{~nm}$ is a critical sampling interval throughout the $0.4-2.45-\mu \mathrm{m}$ wavelength region.

The frequency requirements of coverage are related directly to the dynamic nature of the problem to be studied. Water studies hatve the greatest need for high-frequency measurements, vegetation is next, and geological studies are a distant third. The geological requirements concern acquiring data at an optimum time of year for the proper lighting and vegetation cover in the absence of cloud cover. HIRIS will not acquire continuous coverage but rather will sample sites at high spatial and spectral resolution. By pointing crosstrack $\pm 45^{\circ}$, the maximum time between acquisition for any point on the Earth, except the poles, is 2 days. In some cases. data acquisition is possible on subsequent days even at the Equator.

Pointing is, therefore. a necessary requirement. Nol only is crosstrack pointing required, but an additional requirement exists for along-track pointing in order to develop bidirectional reflectance distribution functions for surfaces, to remove atmospheric absorption and path radiance components, to avoid sun glint on water targets. 


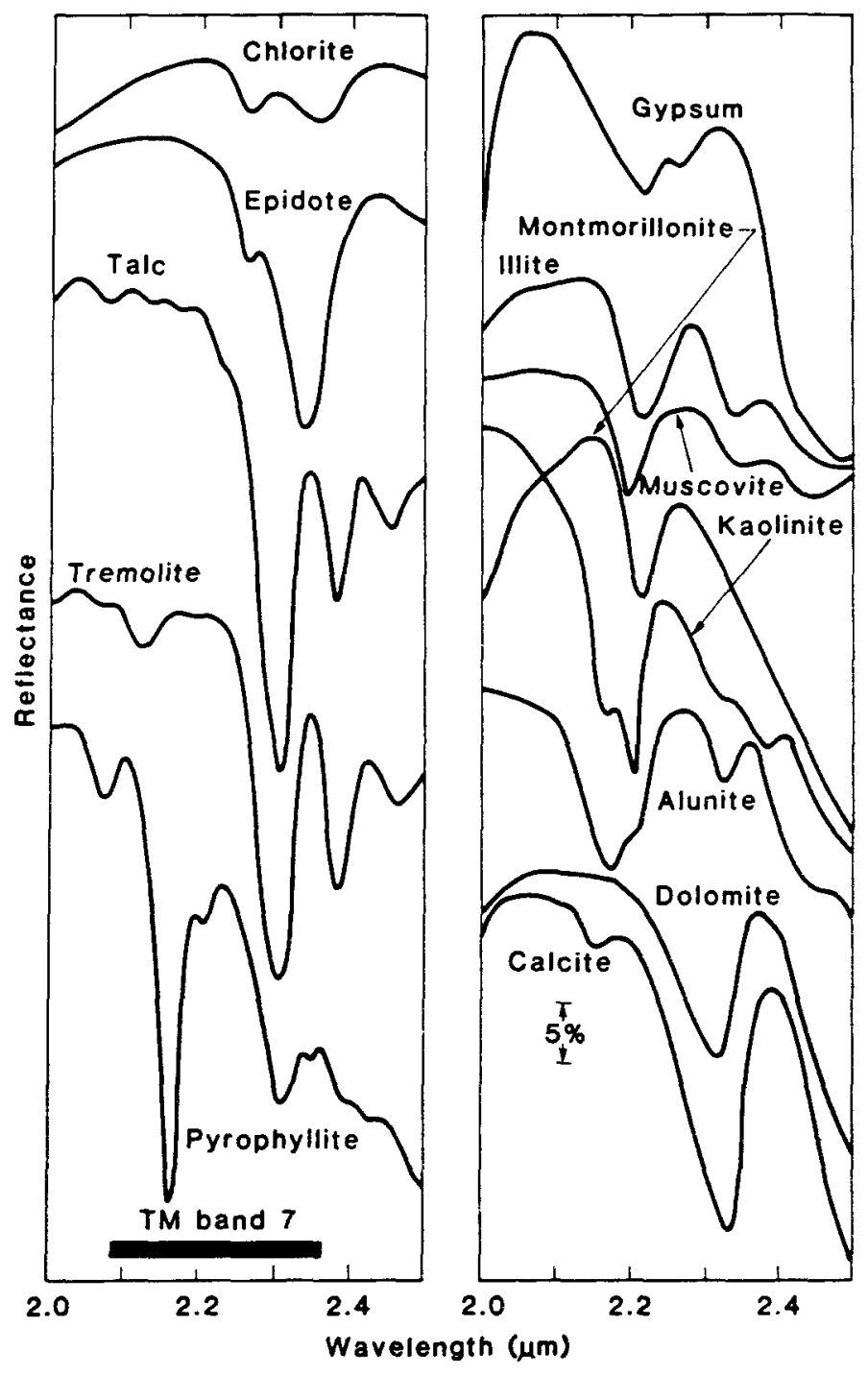

Figure 2. - Laboratory reflectance spectra of common minerals in the 2.0-2.5 $\mathrm{mm}$ region (taken from Goetz and Rowan, 1981).

and to facilitate target tracking in order to increase signal-to-noise performance for dark targets, such as water and any target in the longer wavelength part of the spectrum where the solar irradience is low.

\section{Data analysis}

Over 50 science data products, that is. biogeophysical parameters that can be derived from HIRIS data, have been identified by the HIRIS science team. Here are several examples of the kinds of information that can be derived from HIRIS data, as well as the techniques that are needed for analysis.

\section{Atmospheric water vapor}

HIRIS data will be acquired through the Earth's atmosphere, and because imaging spectrometry data have a much higher spectral resolution than multispectral data scanners such as TM have, much

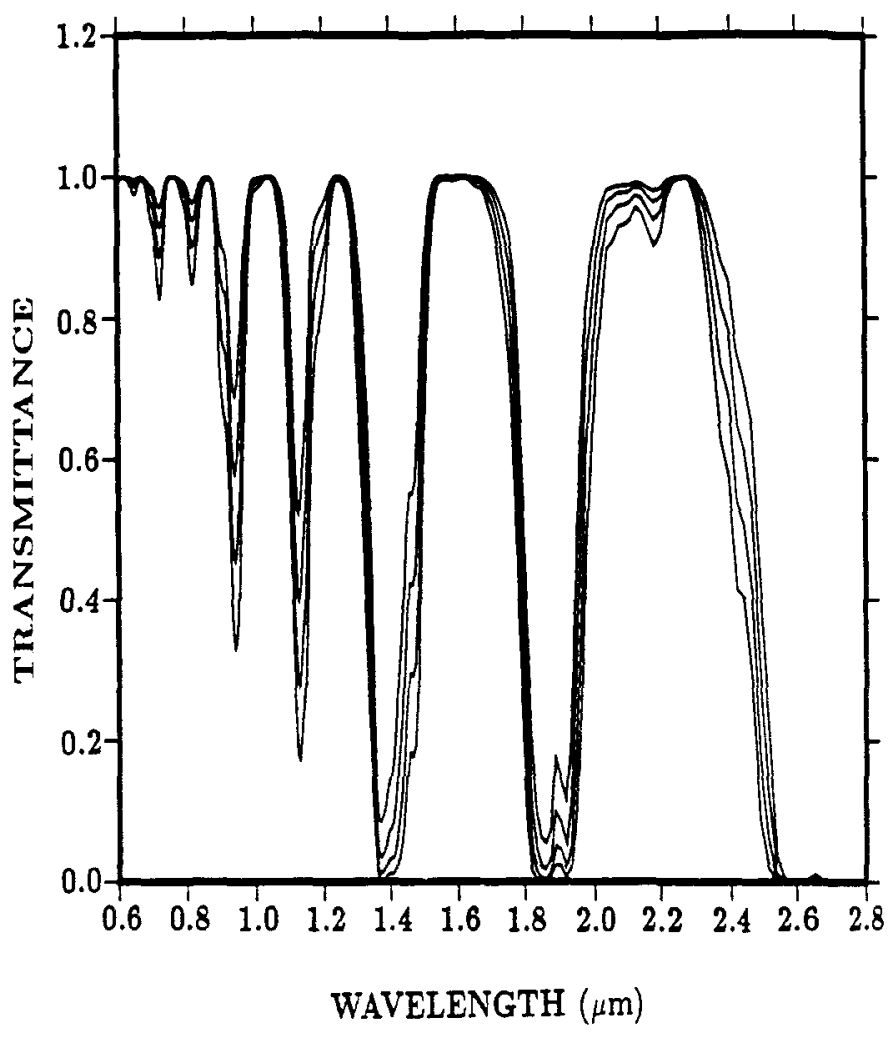

Figure 3.-Vertical atmospheric transmittance as a function of different water vapor amounts. The four curves from top to bottom correspond to column vapor amounts of $0.63,1.3,2.5$, and $5.0 \mathrm{~cm}$, respectively (taken from Gao and Goetz, 1990).

more effort must be applied in order for us to understand and compensate for atmospheric transmission and scattering. In the $0.4-2.5 \mu \mathrm{m}$ region, water vapor absorption affects more than onehalf the spectrum (fig. 3). Carbon dioxide, oxygen, and methane are additional absorbers that have a significant effect in this wavelength region. Initial work has been carried out in deriving column atmospheric water vapor amounts on a pixel by pixel basis, which is necessary in order to remove the variable amounts of water vapor over the scene.

Column atmospheric water vapor amounts at high spatial resolution have been derived from spectral data that was collected by AVIRIS, which covers the spectral region from 0.4 to $2.5 \mu \mathrm{m}$ in $10-\mathrm{nm}$ bands and has a ground instantaneous field of view of $20 \times 20$ $\mathrm{m}$ from an altitude of $20 \mathrm{~km}$ (Gao and Goetz, 1990). The quantitative derivation is made by curve fitting the observed spectra with the calculated spectra in the $1.14-\mu \mathrm{m}$ and $0.94-\mu \mathrm{m}$ water vapor band spectral model by the use of a nonlinear least squares fitting technique. For this analysis, it is mandatory that data be collected in narrow, contiguous spectral bands throughout the region. Figure 4 shows an example of curve fitting of the spectra measured over an unvegetated area.

This spectral matching technique is directly applicable to retrieving column water vapor amounts from AVIRIS spectra, and from HIRIS as well, that are measured on clear days having a visibility of $20 \mathrm{~km}$ or greater. The precision of the retrieved column water vapor amounts from several data sets is 5 percent or better (Gao and Goetz, 1990). Column water vapor images can be constructed by this technique, and they show high variability in the 


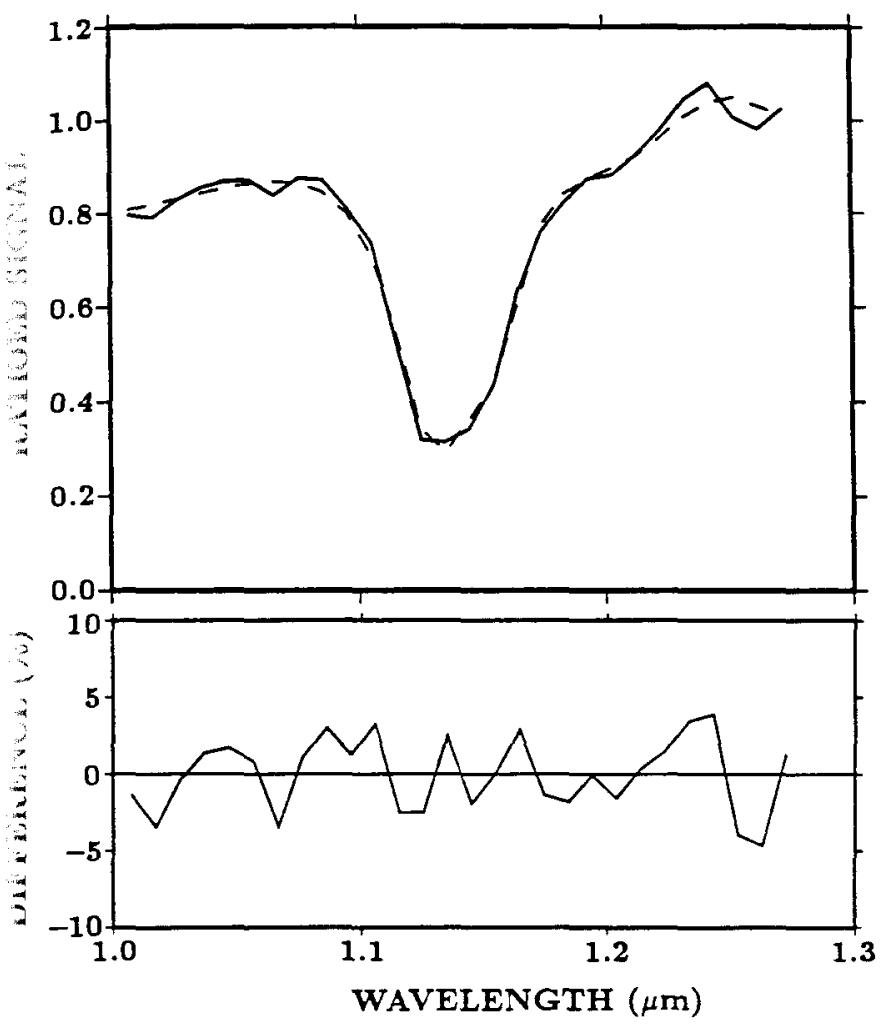

igure 4. - An example of curve fitting of spectra measured over a onvegetated surface. The top plot shows the observed spectrum olid) and the fitted spectrum (dashed). The bottom plot shows the ercent differences between the observed and fitted spectra (taken om Gao and Goetz, 1990).

dantity of water vapor, primarily because of altitude changes, cloud sight, and frontal activity (Gao and Goetz. 1991: Gao and others, in ress).

\section{egetation}

: key contribution of imaging spectrometry to vegetation and ecolgy studies is the quantification of vegetation biochemistry. Estimaon of biochemical constituents in vegetation. such as lignin, celluse, starch, sugar, and protein, by remote sensing methods is an nportant goal in ecological research. The spectral reflectances of ried leaves exhibit diagnostic absorption features that can be used to stimate the abundance of important constituents. Lignin and nitroon concentrations have been obtained from canopies by the use of naging spectrometry and multiple linear regression techniques Wessman and others, 1988). The difficulty in identifying individual pectra of leaf constituents in the region beyond $1 \mu \mathrm{m}$ is that liquid ater contained in the leaf dominates the spectral reflectance of zaves in this region. By means of spectral matching techniques, as iscussed above for determining atmospheric water vapor, we have veen able to remove the liquid water contribution to the spectrum Goetz and others. 1990). The residual spectrum resembles spectra or cellulose in the $1.1 \mu \mathrm{m}$ region, lignin in the $1.7 \mu \mathrm{m}$ region, and ellulose plus starch in the $2.0-2.3 \mu \mathrm{m}$ region (fig. 5). In the entire $1.0-2.3 \mu \mathrm{m}$ region, each of the major constituents contributes to the pectrum. The quantitative estimates will require using unmixing echniques on the residual spectra.
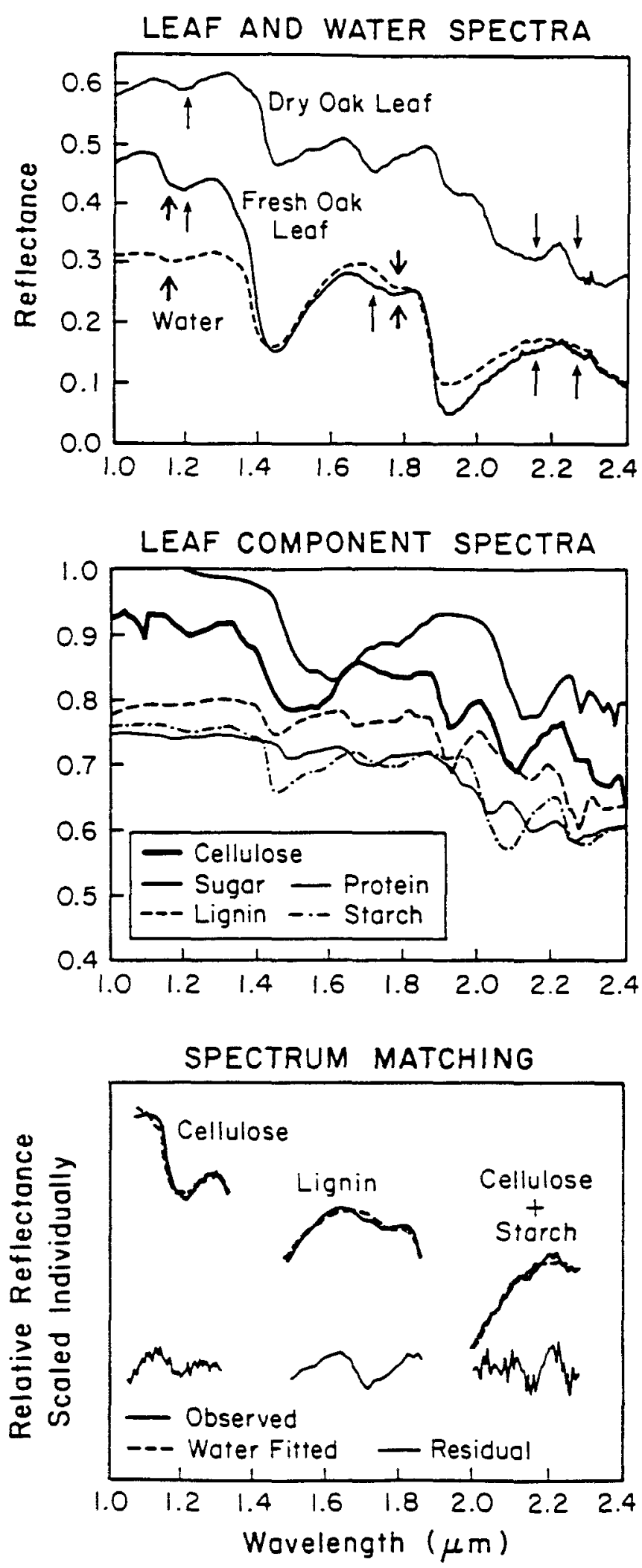

Figure 5.-Reflectance of dry and fresh oak leaves, spectra of leaf biochemical components, and water and residual spectra after water removal (taken from Goetz and others, 1990). 


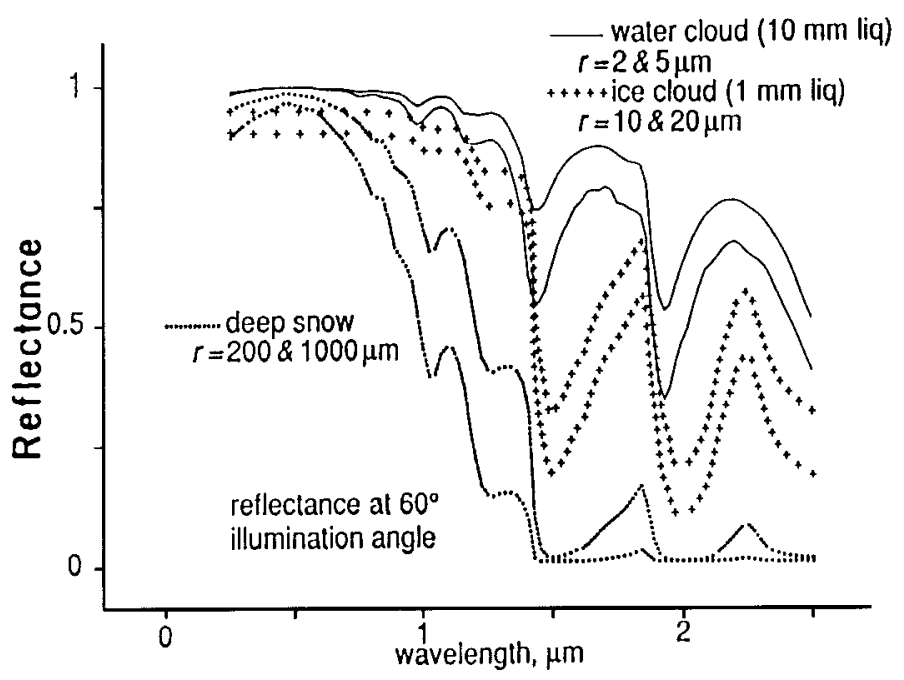

Figure 6. - Spectral signatures of snow, water clouds, and ice clouds. Increased absorption in the 1- to 2- $\mu \mathrm{m}$ range as grain size increases makes it possible to estimate the snow grain size and to separate snow from clouds. Abbreviations: $r$, reflectance; liq, liquid.

\section{Snow and ice}

The extent of snow and ice cover changes dramatically with the season, and as much as 30 percent of the Earth's surface is covered during the Northern Hemisphere's winter. Because snow is highly reflective in the visible and near-infrared region, this has a large effect on the Earth's albedo. Snow albedo depends on grain size, illumination angle, contaminants, and when the pack is thin, depth (Dozier, 1989). Grain size can be measured by using the full spectral resolution of HIRIS (fig. 6). In the near-infrared region, ice is moderately absorptive, and grain size has a large effect on albedo in the 1 - to $1.3-\mu \mathrm{m}$ range.

Contaminants increase absorption in the visible spectrum where snow is highly transparent. In fact, we may be able to identify the type of contaminant by its spectral signature in the visible region. Similarly in a thin snow pack, the spectral signature of the Earth beneath the snow will be observable in the visible region. Finally, the illumination angle is calculated from the solar zenith angle and the topography of the scene. Thus, HIRIS should be ideal for making detailed measurements of snow surface characteristics. A combination of these measurements and the large-scale measurements of MODIS should make it possible to track the energy balance of the snow pack through time.

A second reason for monitoring the snow pack is to estimate the snow melt for water supply and water chemistry properties. The water content is estimated from grain size, depth, and areal extent of the snow pack. The melt rate is estimated from these parameters and an estimate of the amount of energy absorbed by the snow pack. This can be calculated from the albedo measurements.

\section{Image processing}

The data sets produced by imaging spectrometry (fig. 7) are large and not easy to manipulate by using today"s available software. Each $10 \times 10-\mathrm{km}$ AVIRIS image contains approximately 140 megabytes of data. However, new developments have made it possible to interact with AVIRIS data and to carry out analyses, such as those discussed above, on RISC work stations (F. Kruse, oral communication,
1991). The Spectral Image Processing System is now available from the University of Colorado, Boulder, Colorado, USA.

\section{Summary}

HIRIS is designed to acquire images in 192 spectral bands simultaneously throughout the solar-reflective part of the spectrum. It will provide the link between measurements of processes at the human scale and global monitoring by MODIS. HIRIS data are applicable to many disciplines in the earth sciences and will be particularly valuable in detecting change on land and in the bordering oceans.

Data analysis techniques include the powerful tools of spectrum matching, which so far have provided unique results in the determination of column atmospheric water vapor amounts at high spatial resolution, as well as in vegetation biochemistry. The data sets are large but rich in information.

\section{Acknowledgments}

The author wishes to thank Dave Norris of the Jet Propulsion Laboratory (JPL), HIRIS project manager during the design development phase, for his many ideas and innovations, including image motion compensation. Thanks also go to the many other JPL personnel, including Don Rockey, Deb Vane, Valerie Duval, Fred Vescelus, Terry Reilly, Mark Herring, and Teri Smith. At the University of Colorado, Bo-Cai Gao developed and applied the concepts of spectrum matching. Last but not least, the HIRIS science team members contributed to the wording for the justification in their various disciplines. The part of the research described in this article was carried out at the University of Colorado and was supported by contract number 958039 with the Jet Propulsion Laboratory and NAS5-30552 with the Goddard Space Flight Center, Greenbelt, Maryland, USA.

\section{References}

Abrams, M.J., Ashley, R.P., Rowan, L.C., Goetz, A.F.H., and Kahle, A.B., 1977, Mapping of hydrothermal alteration in the Cuprite mining district, Nevada, using aircraft scanner images for the spectral region 0.46 to $2.36 \mu \mathrm{m}$ : Geology, v. 5, p. 713-718.

Collins, W., Chang. S.H., Raines, G., Canney, F., and Ashley, R., 1983. Airborne biogeophysical mapping of hidden mineral deposits: Economic Geology, v. 78, p. 737-749.

Dozier, J., 1989, Spectral signature of alpine snow cover from the Landsat Thematic Mapper: Remote Sensing of Environment, v. 28, p. 9-22.

Gao, B.C., and Goetz, A.F.H., 1990, Column atmospheric water vapor retrievals from airborne imaging spectrometer data: Journal of Geophysical Research, v. 95D, p. 3549-3564.

1991, Cloud area determination from AVIRIS data using water vapor channels near $1 \mu \mathrm{m}$ : Journal of Geophysical Research, v. 96D, p. $2857-2864$

Gao, B.C., Westwater, E.R., Stankov, B.B., Birkenheuer, D. and Goetz, A.F.H., in press, Comparison of column water vapor measurements using downward-looking optical and infrared imaging systems and upward-looking microwave radiometer: Journal of Applied Meteorology.

Goetz, A.F.H., 1987, The Portable Instant Display and Analysis Spectrometer (PIDAS), in Airborne Imaging Spectrometer Data Analysis Workshop, 3rd. Pasadena, California, USA, 1987, Proceedings: JPL Publication 87-30, p. 8-17.

Goetz, A.F.H., Billingsley, F.C., Elston, D., Lucchitta, I., Shoemaker, E.M., Abrams, M.J., Gillespie, A.R., and Squires, R.L., 1975, Application of ERTS images and image processing to regional geologic problems and geologic mapping in northern Arizona: JPL Technical Report TR-32-1597, $188 \mathrm{p}$. 


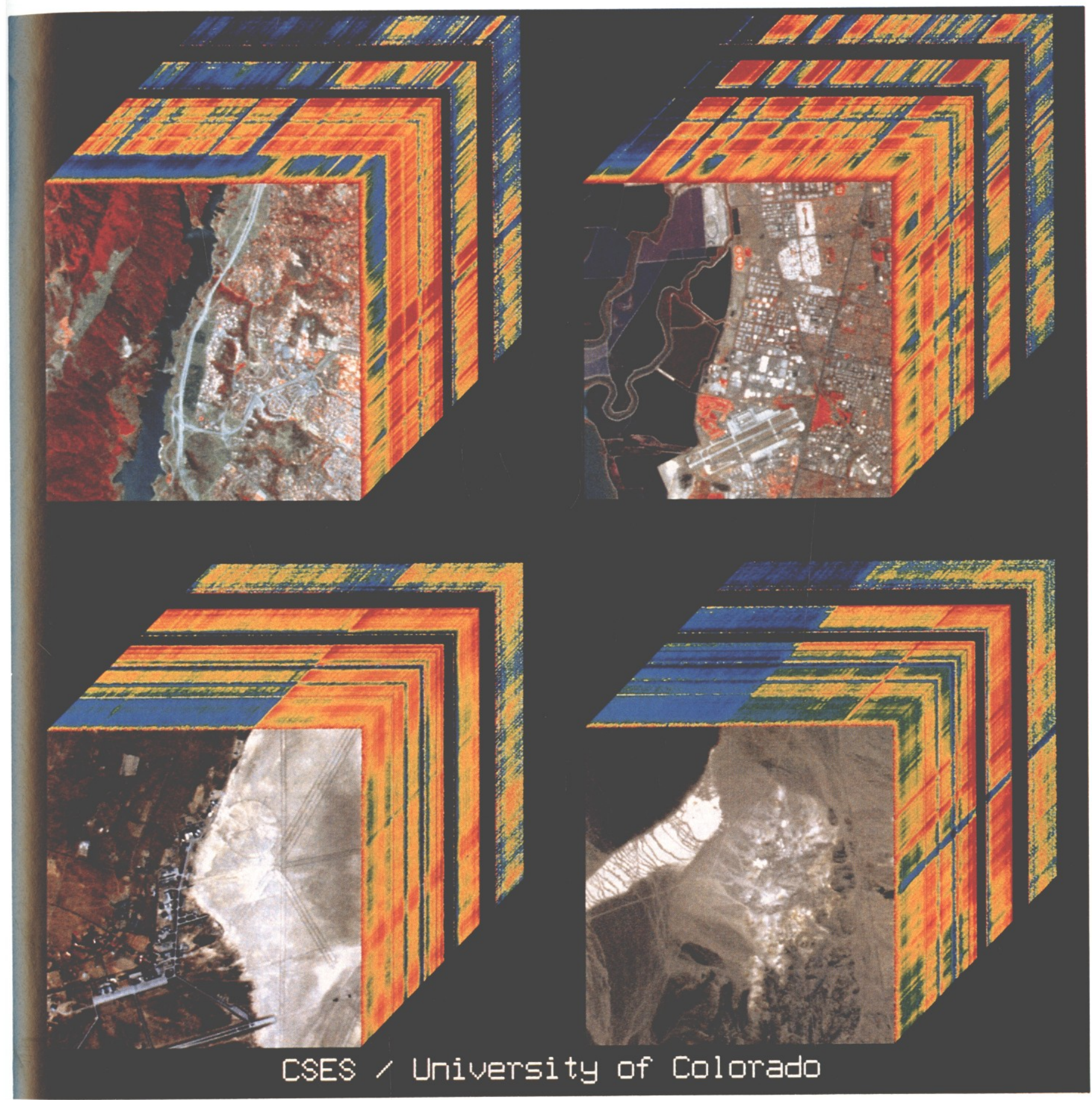

Figure 7.-AVIRIS image cubes. Each represents a stacked set of 224 spectral band images. The front face of the cube is a color infrared composite made from three spectral band images, and the sides of the cube represent the color-coded surface radiance of each of the edge pixels. The wavelength ranges from $0.4 \mu \mathrm{m}$ at the front to $2.45 \mu \mathrm{m}$ at the back. Red is high and blue is low, whereas the dark bands represent the atmospheric water vapor absorption bands at 1.4 and $1.9 \mu \mathrm{m}$. 
Goetz, A.F.H., Gao, B.C., Wessman, C.A., and Bowman, W.D., 1990, Estimation of biochemical constituents from fresh, green leaves by spectrum matching techniques: International Geoscience and Remote Sensing Symposium, Remote Sensing Science for the Nineties (IGARSS '90), College Park, Maryland, USA, 1990, Proceedings, v. 2, p. 971-974.

Goetz, A.F.H., and others, 1991, HIRIS science requirements document: Pasadena, California, USA, Jet Propulsion Laboratory, JPL D-7843 (JPL internal document), $25 \mathrm{p}$.

Goetz, A.F.H., and Rowan, L.C., 1981, Geologic remote sensing: Science, v. 211, p. $781-791$.

Goetz, A.F.H., Rowan, L.C., and Kingston, M.J., 1982, Mineral identification from orbit: Initial results from the Shuttle Multispectral Infrared Radiometer: Science, v. 218, p. 1020-1024.

Goetz, A.F.H., Vane, G., Solomon, J., and Rock, B.N., 1985, Imaging spectrometry for Earth remote sensing: Science, v. 228, p. 1147-1153.

Hunt, G.R., 1980, Electromagnetic radiation: The communication link in remote sensing, in Siegal, B.S., and Gillespie, A.R., eds., Remote sensing in geology: New York, John Wiley, p. 5-45.

Porter, W.M., and Enmark, H.T., 1988, System overview of the Airborne Visible/Infrared Imaging Spectrometer (AVIRIS), in Vane, Gregg, ed., Imaging Spectroscopy II, San Diego, California, USA, 1987: SPIE [Society of Photo-Optical Instrumentation Engineers] Proceedings, v. 834 , p. $22-31$.

Vane, G., and Goetz, A.F.H., 1988, Terrestrial imaging spectroscopy: Remote Sensing of Environment, v. 24, p. 1-29.

Wessman, C.A., Aber, J.D., Peterson, D.L., and Melillo, J.M., 1988, Remote sensing of canopy chemistry and nitrogen cycling in temperate forest ecosystems: Nature (London), v. 335, p. 154-156.

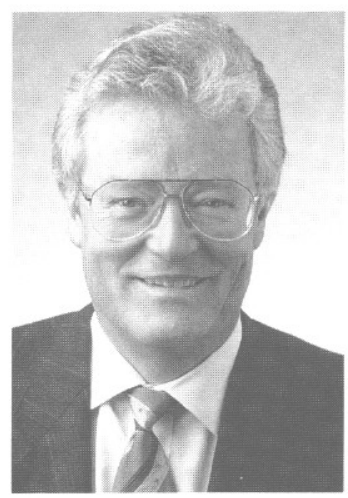

Dr. Alexander F.H. Goetz is a Professor of Geological Sciences and Director of the Center for the Study of Earth from Space (CSES) within the Cooperative Institute for Research in Environmental Sciences (CIRES). He received his degrees from the California Institute of Technology in the USA. Prior to joining the University of Colorado in 1985, he spent 15 years at the NASA Jet Propulsion Laboratory, where he was a Senior Research Scientist and Manager of the Imaging Spectrometer Program. Since 1989 he has been Team Leader for NASA's Earth Observing System (EOS), High Resolution Imaging Spectrometer (HIRIS) facility. 